\title{
Spontaneous Coronary Artery Dissection
}

\author{
Jacqueline Saw
}

Division of Cardiology, Vancouver General Hospital, University of British Columbia, Vancouver, British Columbia, Canada

\begin{abstract}
Spontaneous coronary artery dissection (SCAD) is a non-traumatic and non-iatrogenic separation of the coronary artery wall that is now recognised as an important cause of myocardial infarction, especially in younger women. SCAD can be elusive on coronary angiography and clinician familiarity with non-pathognomonic angiographic SCAD variants and the use of intracoronary imaging will improve diagnosis. Conservative management and long-term cardiovascular follow-up are typically recommended.
\end{abstract}

\section{Keywords}

Myocardial infarction, non-atherosclerotic, spontaneous coronary artery dissection

Disclosure: Dr. Saw has received unrestricted research funding (Canadian Institutes of Health Research, University of British Columbia Division of Cardiology, AstraZeneca, Abbott Vascular, St Jude Medical, Boston Scientific, Servier), speaker honoraria (AstraZeneca, St Jude Medical, Boston Scientific, Bayer, Sunovion), consultancy and advisory board honoraria (AstraZeneca, St Jude Medical, Boston Scientific, Abbott Vascular), and proctorship honoraria (St Jude Medical, Boston Scientific).

Received: 12 August 2015 Accepted: 13 August 2015 Citation: Interventional Cardiology Review, 2013;10(3):142-3

Correspondence: Jacqueline Saw, MD, FRCPC, FACC, FSCAI, Interventional Cardiology Clinical Associate Professor, 2775 Laurel Street, Level 9, Vancouver General Hospital, Vancouver, BC, V5Z1M9, Canada. E: jsaw@mail.ubc.ca

Spontaneous coronary artery dissection (SCAD) is increasingly recognised as an important cause of myocardial infarction (MI), especially in younger women. It is defined as a non-traumatic and non-iatrogenic separation of the coronary artery wall, which creates a false lumen that may or may not be in continuity with the true lumen. Although conventionally SCAD may be atherosclerotic or non-atherosclerotic in origin, contemporary series have focused on the non-atherosclerotic variant, since the pathophysiology, management, and outcomes are distinct from atherosclerotic coronary artery disease (which causes atherosclerotic SCAD). As such, contemporary usage of the term 'SCAD' is typically synonymous with non-atherosclerotic SCAD.

SCAD may be a result of an intimal-medial tear that can manifest as multiple radiolucent lumen on angiography, or it may result from spontaneous haemorrhage into the arterial wall that can be angiographically subtle. In fact, $<30 \%$ of SCAD have angiographic type 1 appearance (pathognomonic contrast stain of arterial wall with multiple radiolucent lumen) in the author's large contemporary series. ${ }^{1}$ Thus, intracoronary imaging with optical coherence tomography (OCT) or intravascular ultrasound (IVUS) play important roles in the diagnosis of non-type 1 angiographic variant of SCAD. These type 2 and 3 variants have angiographic appearance of diffuse smooth stenosis or mimic atherosclerosis, respectively. ${ }^{2}$ The diagnosis of SCAD on OCT or IVUS requires presence of intramural haematoma and/or separation of the intimomedial membrane creating double lumen. ${ }^{3}$ In the author's largest reported intracoronary imaging SCAD series of 22 patients, the lengths of these dissections on angiography are long, especially the type 2 forms (mean length $~ 58 \mathrm{~mm}$ ). ${ }^{3}$ These angiographic variants were previously poorly recognised, resulting in missed and misdiagnosis of SCAD. Thus, there is a great emphasis on angiographers to become familiar with these non-pathognomonic angiographic SCAD variants to improve diagnosis.
The etiology of SCAD is not fully understood, but recent large series suggest that the majority of patients have potential underlying predisposing arteriopathy, such as fibromuscular dysplasia (FMD), connective tissue disorder and systemic inflammatory conditions, or the condition may be pregnancy-related.' Less than $20 \%$ may be idiopathic, where no predisposing arteriopathy is identified after vascular screening or detailed questionnaires for these disorders. The most dominant association with SCAD is FMD. Since the author's discovery of the strong association and publication of the first case series of concomitant SCAD and FMD in $2012,{ }^{4}$ the author and others subsequently reported that non-coronary FMD was present in up to $86 \%$ of patients with SCAD. 15,6 On the other hand, pregnancy-related SCAD (previously thought to be a major cause of SCAD) has a much lower reported frequency $(<5 \%)$ as a predisposing cause in contemporary series. ${ }^{7}$ Additional precipitating stressors, such as intense emotional stress, physical activities, hormone therapy, sympathomimetic drugs and intense valsalva-like activities (e.g. vaginal delivery, coughing, retching, vomiting, bowel movement) have been reported to precipitate SCAD, ${ }^{1}$ especially in patients with underlying predisposing arteriopathy.

The management of SCAD remains relatively unexplored. There are no randomised trials comparing conservative treatment with revascularisation. Furthermore, standard medications administered for cardiac patients have not been studied in the SCAD population. Thus, recommendations on SCAD management remain empiric and largely based on expert opinion. The rationale and strategy of pharmaceutical therapy for SCAD was previously reviewed in detail. ${ }^{8}$ In essence, aspirin and beta-blocker are administered long-term for secondary thrombotic prevention and reduction of arterial shear-stress, respectively. ACE inhibitor is administered for patients with left ventricular dysfunction and statins for patients with underlying dyslipidemia. In terms of revascularisation, the expert consensus is conservative management 
unless patients have ongoing/recurrent ischaemia, haemodynamic instability, or left main dissection, since invasive management with coronary stenting is often challenging and can have suboptimal results (procedural success in 50-70 \%). ${ }^{19}$ The results of long-term outcome with bypass grafting are inconclusive as they are derived from small case series of $<15$ patients; nevertheless, there is concern over graft patency with one study showing $75 \%$ of grafts occluded at follow-up. ${ }^{10}$

Recent series of prospectively followed SCAD patients have provided important information on acute and long-term cardiovascular outcomes. Most patients present with acute coronary syndromes with either nonST elevation or ST-elevation MI and a small proportion have ventricular arrhythmias. Acute in-hospital mortality was relatively low $(<5 \%)$ in contemporary series. Recurrent MI or need for revascularisation in initially conservatively managed patients was $5-10 \% .{ }^{1,10}$ However, following the acute event, a significant proportion of patients have recurrent chest pains (often atypical in features). Subacute major adverse cardiovascular events (MACE) were reported at 10-20\% at 2-year follow-up, with recurrent SCAD event of 15\%. At longer-term follow-up from retrospective cohorts, estimated rate of MACE can be up to $50-60 \% .^{1,10}$ Therefore, SCAD survivors should be closely followed by cardiovascular specialists.

In summary, SCAD can be elusive on coronary angiography and clinicians should become more cognisant of non-pathognomonic angiographic SCAD variants and consider intracoronary imaging to improve diagnosis. Conservative management is typically recommended unless patients have ongoing ischaemia, haemodynamic instability or left main involvement. Long-term cardiovascular follow-up is important since recurrent events frequently occur post-SCAD.
1. Saw J, Aymong E, Sedlak T, et al. Spontaneous coronary artery dissection: association with predisposing arteriopathies and precipitating stressors and cardiovascular outcomes. Circ Cardiovasc Interv 2014;7:645-55.

2. Saw J. Coronary angiogram classification of spontaneous coronary artery dissection. Catheter Cardiovasc Interv 2014;84:1115-22

3. Saw J, Mancini GB, Humphries K, et al. Angiographic appearance of spontaneous coronary artery dissection with intramural hematoma proven on intracoronary imaging. Catheter Cardiovasc Interv 2015:epub ahead of print.
4. Saw J, Poulter R, Fung A, et al. Spontaneous coronary artery dissection in patients with fibromuscular dysplasia: a case series.Circ Cardiovasc Interv 2012;5:134-7.

5. Saw J, Ricci D, Starovoytov A, et al. Spontaneous coronary artery dissection: prevalence of predisposing conditions including fibromuscular dysplasia in a tertiary center cohort. JACC Cardiovasc Interv 2013;6:44-52.

6. Prasad M, Tweet MS, Hayes SN, et al. Prevalence of extracoronary vascular abnormalities and fibromuscular dysplasia in patients with spontaneous coronary artery dissection. Am J Cardiol 2015;115:1672-7.
7. Vijayaraghavan $\mathrm{R}$, Verma $\mathrm{S}$, Gupta $\mathrm{N}$, Saw Pregnancy-related spontaneous coronary artery dissection. Circulation 2014;130:1915-20.

8. Saw J. Spontaneous coronary artery dissection. Can J Cardiol 2013;29:1027-33.

9. Tweet MS, Eleid MF, Best PJ, et al. Spontaneous coronary artery dissection: revascularization versus conservative therapy. Circ Cardiovasc Interv 2014;7:777-86.

10. Tweet MS, Hayes SN, Pitta SR, et al. Clinical Features, Management and Prognosis of Spontaneous Coronary Artery Dissection. Circulation 2012;126:579-88. 